\title{
The Use of Dichloroacetate in the Treatment of Overwhelming Hypoxic Acidosis
}

\author{
Joyce A. Wahr, MD, Katherine Ullrich, and Steven F. Bolling, MD
}

\begin{abstract}
Overwhelming hypoxic acidosis due to poor tissue oxygen delivery from low cardiac output, pulmonary failure, and other causes has devastating effects postoperatively on patient outcome. Whereas conventional therapeutics often can not reverse the downward spiral of these patients, dichloroacetate (DCA) has been shown to be beneficial. This study investigated the metabolic and hemodynamic effects of DCA given after the onset of overwhelming hypoxic acidosis in a canine model. A hypoxically ventilated canine model of severe induced acidosis was established and dogs surviving the development of acidosis were randomized to receive DCA or sodium chloride $(\mathrm{NaCl})$ treatment. Dogs receiving DCA after development of hypoxic lactic acidosis showed no further change in metabolic parameters during the 90-minute treatment period $\left(\mathrm{pH}, 7.24\right.$ to $7.23 ; \mathrm{HCO}_{3}, 17.7$ to $18 \mathrm{mmol} / \mathrm{L}$; lactate, 2.04 to $1.05 \mathrm{mM} / \mathrm{L}$ ); whereas animals receiving an equivalent sodium load showed progressive, significant deterioration in all parameters $(\mathrm{pH}, 7.24$ to 7.12;
\end{abstract}

$\mathbf{A}$ LTHOUGH LACTIC acidosis may occur when tissue oxygenation is normal (hepatic and renal failure, diabetes, malignancy, or toxins), it most frequently occurs from tissue hypoxia. The development of lactic acidosis due to postoperative low cardiac output, pulmonary failure, sepsis, or shock and the ensuing tissue hypoxia carries an ominous prognosis with a patient mortality greater than $85 \% .^{1-3}$ In postoperative patients with severe low output, plasma lactate levels may rise fivefold, $\mathrm{pH}$ decreases below 7.25 , and conventional therapy fails to break the downward spiral that ensues. The efficacy of conventional therapy, including catecholamines and sodium bicarbonate, has been questioned, as recent animal studies suggest that such therapy may actually increase lactate production, thereby resulting in decreased cardiac output. ${ }^{4-7}$

Sodium dichloroacetate (DCA) is a carboxylic acid that alters the ability of cells to use lactate as substrate and has been shown to have beneficial metabolic and hemodynamic effects in lactic acidosis. ${ }^{8-10}$ DCA has been shown to decrease canine mortality in phenformin and hepatectomyrelated lactic acidosis, ${ }^{9}$ lower lactate, and raise $\mathrm{pH}$ during hypoxia in dogs and rats ${ }^{11,12}$ and improve blood pressure in a hypoxic rat model..$^{12}$ Clinically, DCA has returned serum $\mathrm{pH}$ and bicarbonate levels towards normal and improved arterial perfusion ${ }^{13,14}$ in patients with lactic acidosis and multi-organ failure.

DCA may have beneficial cardiac effects and has been noted to limit ST segment elevation and myocardial lactate release associated with coronary artery occlusion, ${ }^{15}$ enhance myocardial function in endotoxin-shocked rats, ${ }^{16}$ and

From the Departments of Anesthesiology and Surgery (Thoracic Surgery), The University of Michigan, Ann Arbor, MI.

Supported in part by the American Heart Association of Michigan.

Address reprint requests to Joyce A. Wahr, MD, Department of Anesthesiology, IG323 0048, 1500 E Medical Center Dr, Ann Arbor, MI 48109-0344.

Copyright $\odot 1994$ by W.B. Saunders Company

1053-0770/94/0801-0014\$03.00/0
$\mathrm{HCO}_{3}, 16.8$ to $13.2 \mathrm{mM} / \mathrm{L}$; lactate, 2.05 to $3.55 \mathrm{mM} / \mathrm{L}$. Myocardial blood flow was significantly increased by hypoxia in all dogs. Finally, cardiac output and stroke volume were significantly increased at 90 minutes by DCA versus control. Myocardial oxygen utilization efficiency (LV work / M $\mathrm{VO}_{2}$ ) was improved during DCA treatment. DCA, a carboxylic acid, increases pyruvate dehydrogenase activity, thereby enhancing lactate use as a metabolic substrate. DCA had an ameliorative metabolic effect, and benefitted myocardial performance without a direct inotropic effect. DCA treatment appears to enhance myocardial performance on a metabolic and not primarily inotropic basis, does not increase the "cost" of myocardial work, and warrants further study. Copyright $\odot 1994$ by W.B. Saunders Company

KEY WORDS: hemodynamics, myocardial oxygen utilization, myocardial ischemia

improve myocardial efficiency and lactate use in patients with heart failure. ${ }^{17}$ The hemodynamic and metabolic effects of DCA were studied in a canine model of severe hypoxic lactic acidosis to determine if DCA enhanced myocardial performance primarily or secondarily from the metabolic improvement seen with DCA therapy.

\section{MATERIALS AND METHODS}

Mongrel dogs (16 to $22 \mathrm{~kg}$ ) were anesthetized with $30 \mathrm{mg} / \mathrm{kg}$ of pentobarbital, endotracheally intubated and ventilated to maintain a $\mathrm{PaCO}_{2}$ of 35 to $45 \mathrm{mmHg}$ throughout the experiment. Each dog received additional pentobarbital ( 50 to $100 \mathrm{mg} / \mathrm{h}$ ) as needed to achieve adequate anesthesia, $1 \mathrm{mg} / \mathrm{kg}$ pancuronium initially, and 1 to $2 \mathrm{mg} / \mathrm{h}$ to maintain paralysis. Sodium bicarbonate was administered initially to achieve a normal $\mathrm{pH}$ and correct any base deficit prior to baseline measurements. Sodium chloride $(0.9 \%)$ was administered to each dog to achieve a pulmonary capillary wedge pressure (PCWP) of 5 to $10 \mathrm{mmHg}$ at baseline. This was continued at 4 to $6 \mathrm{~mL} / \mathrm{kg} / \mathrm{h}$ for the duration of the experiment.

The left femoral and right carotid arteries were catheterized with Tygon (Norton, Akron, OH) tubing (0.04 mm I.D.) for simultaneous withdrawal of arterial samples during microsphere injection. Mean arterial pressure (MAP) was measured with a Statham P23dB transducer (Gould, Akron, $\mathrm{OH}$ ) connected to a catheter inserted into the right carotid artery. A pulmonary artery catheter was placed via the right internal jugular vein to monitor pulmonary artery pressure (PAP) and PCWP. Cardiac output (CO) was determined in triplicate by thermodilution using $10 \mathrm{~mL}$ of iced saline. A Millar high fidelity micromanometer (model PC350, Houston, TX) catheter was placed via a carotid artery retrograde into the left ventricle (LV) to measure LV pressures. The LV pressure signal was differentiated electronically to continuously measure its first derivative, $+\mathrm{dP} / \mathrm{dt}$. A catheter was inserted via the urethra into the bladder to continuously measure urinary output. The inspiratory oxygen concentration was constantly monitored with a Beckman LB 2 medical gas monitor (Fullerton, CA). Arterial blood samples were analyzed using a Radiometer ABL 2 (Copenhagen, Denmark). Serum lactate determinations were made on whole blood using a Yellow Springs Lactate Analyzer (model 23L, Yellow Springs, $\mathrm{OH}$ ). Serum and urine sodium, calcium and potassium determinations were determined using a Nova 6 electro- 
lyte analyzer (Waltham, MA) and blood glucose levels were determined using a Beckman (Fullerton, CA) glucose analyzer 2.

Following induction of anesthesia, a left thoracotomy was performed in the fifth intercostal space and the heart suspended in a pericardial cradle. A Tygon catheter was placed in the left atrium for measurement of left atrial pressure (LAP) and for injection of microspheres. In 8 animals, a 23 -gauge catheter was placed under direct vision in an obtuse marginal epicardial vein on the surface of the LV and tunneled through the skin to permit intermittent sampling of myocardial venous blood for determination of myocardial lactate extraction and oxygen consumption. The chest was then closed using \#2 polypropylene suture to oppose the ribs and 2-0 silk for a two-layer closure of the skin. The lungs were reexpanded with a series of Valsalva maneuvers and normal ventilation was resumed. This protocol was approved by the University of Michigan Institutional Animal Care and Use Committee and complied with the "Principles of Laboratory Animal Care" and the "Guide for the Care and Use of Laboratory Animals" (NIH Publication No. 80.23, revised 1978 ).

Regional myocardial blood flow (MBF) and cortical and medullary renal blood flow were measured with radioactive tracerlabeled microspheres $(15 \mu \mathrm{m}$ in diameter, New England Nuclear, Billerica MA), using the reference withdrawal method. ${ }^{18}$ Five injections were made in each experiment using one of six available isotopes $\left({ }^{141} \mathrm{Ce},{ }^{113} \mathrm{Sn},{ }^{51} \mathrm{Cr},{ }^{103} \mathrm{Ru},{ }^{95} \mathrm{Nb},{ }^{46} \mathrm{Sc}\right)$, chosen at random, for each flow determination. Adequate dispersal of microspheres in their suspension ( 1 to 2 million microspheres per injection) was achieved by sonication for 30 minutes and vortexing for 5 minutes prior to injection into the left atrium. Reference arterial samples were obtained simultaneously from both femoral and carotid arteries at a constant rate $(7.0 \mathrm{~mL} / \mathrm{min})$ with a Harvard withdrawal pump; withdrawals were initiated before microsphere injection and completed 2 minutes later.

Thirty minutes were allowed for the dog to stabilize after completion of instrumentation, during which time the animals were ventilated with an $\mathrm{F}_{1} \mathrm{O}_{2}$ of 1.0. While recording baseline hemodynamic parameters, an injection of microspheres was performed and blood was collected for baseline (BL) metabolic measurements. The $\mathrm{F}_{\mathrm{I}} \mathrm{O}_{2}$ was then gradually lowered to approximately $8 \%$ to $12 \%$, determined in each dog individually as the oxygen percentage that achieved a $\mathrm{PaO}_{2}$ of 25 to $32 \mathrm{mmHg}$, and the animals were ventilated with that $\mathrm{F}_{1} \mathrm{O}_{2}$ for the remainder of the protocol. Arterial blood gases were determined every 5 to 10 minutes to ensure a stable state of hypoxia. The development of hypoxic lactic acidosis was defined by a $\mathrm{pH}$ of $<7.25$ and a $10 \%$ or greater decrease in $\mathrm{HCO}_{3}-$ from baseline. At the time of achieving hypoxic lactic acidosis (HYP 0), hemodynamic and metabolic measurements were performed and a second microsphere injection was made. The time to development of hypoxic lactic acidosis varied between dogs (range 45 to $120 \mathrm{~min}$ ), but was not significantly different between groups. Dogs were then randomly assigned to receive an infusion of DCA, $300 \mathrm{mg} / \mathrm{kg} / \mathrm{h}$, or an infusion of hypertonic saline $(4.4 \% \mathrm{NaCl})$ to equal the sodium content of an equivalent DCA dose. The total volume of fluid administered in both groups was equal. Hemodynamic, metabolic, and microsphere flow measurements were performed every 30 minutes thereafter following the achievement of hypoxic lactic acidosis (HYP 30, HYP 60, HYP 90). In those animals with epicardial vein cannula, 1-mL blood samples were obtained by collecting free-flowing blood from the epicardial vein. Blood was collected under a thin film of mineral oil and then drawn into a heparinized syringe for determination of venous blood gases and lactate determination. Arterial blood gases and lactate levels were also obtained. Myocardial lactate extraction (MLE) was determined by the equation: MLE =

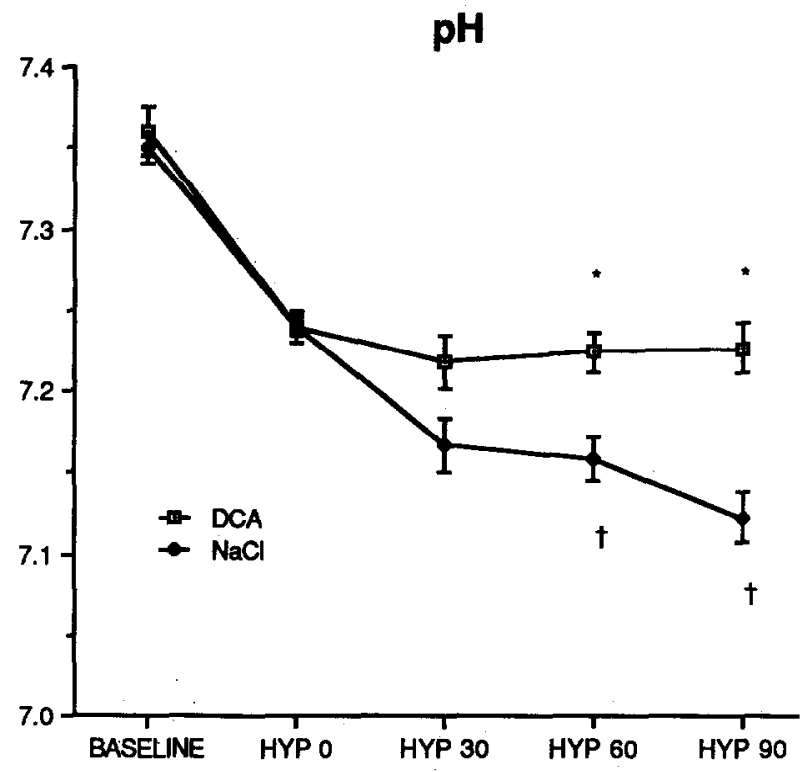

Fig 1. Changes in serum pH during treatment of hypoxic lactic acidosis with DCA or NaCl. All values are mean \pm SEM. $* P<0.01$ compared to $\mathrm{NaCl}$. $+\boldsymbol{P}<0.01$ compared to HYP.

[(arterial lactate - epicardial lactate)/arterial lactate] $\times 100$ and myocardial oxygen consumption $\left(\mathrm{MVO}_{2}\right.$ in $\mathrm{mL} \mathrm{O}_{2} / \mathrm{g}$ tissue $/ \mathrm{min}$ ) by the equation $\mathrm{MVO}_{2}=$ [arterial $\mathrm{O}_{2}$ content-epicardial venous $\left.\mathrm{O}_{2}\right] \times$ [MBF $(\mathrm{mL} / \mathrm{g})]$. Left ventricular work was determined by the equation [MAP-CVP] $\times \mathrm{CO}$ and myocardial oxygen use efficiency was then determined by dividing the work done (LV work) by the oxygen consumption $\left(\mathrm{MVO}_{2}\right)$.

At the end of the experiment, the dogs were sacrificed humanely with intravenous $\mathrm{KCl}$. The heart and kidneys were removed and placed in formalin for sectioning. Multiple full thickness sections of the myocardium were obtained in a standardized fashion around complete rings of the LV. Each block of tissue was divided into three sections of approximately equal thickness from endocardium

\section{SERUM BICARBONATE}

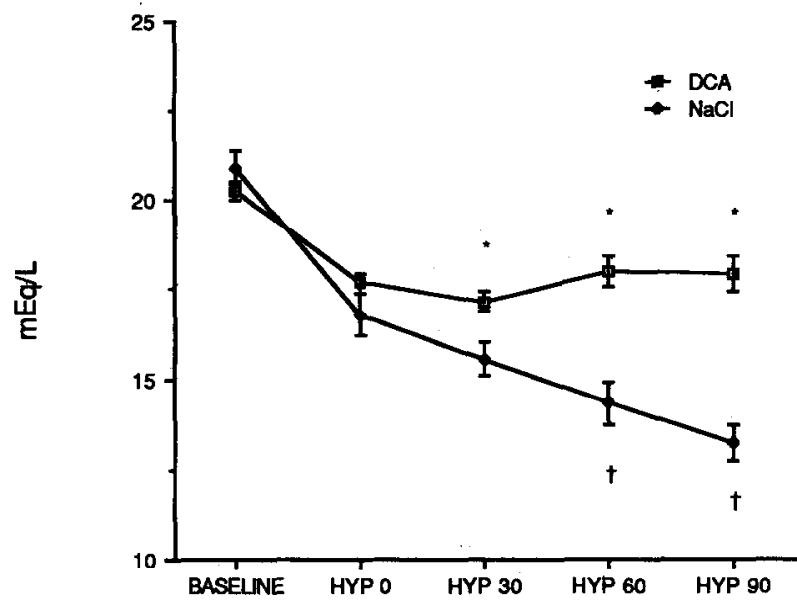

Fig 2. Changes in serum bicarbonate during treatment of hypoxic lactic acidosis with DCA or $\mathrm{NaCl}$. All values are mean $\pm S E M$. $* P<$ 0.01 compared to $\mathrm{NaCl}$. $+P<0.01$ compared to $H Y P$. 


\section{LACTATE}

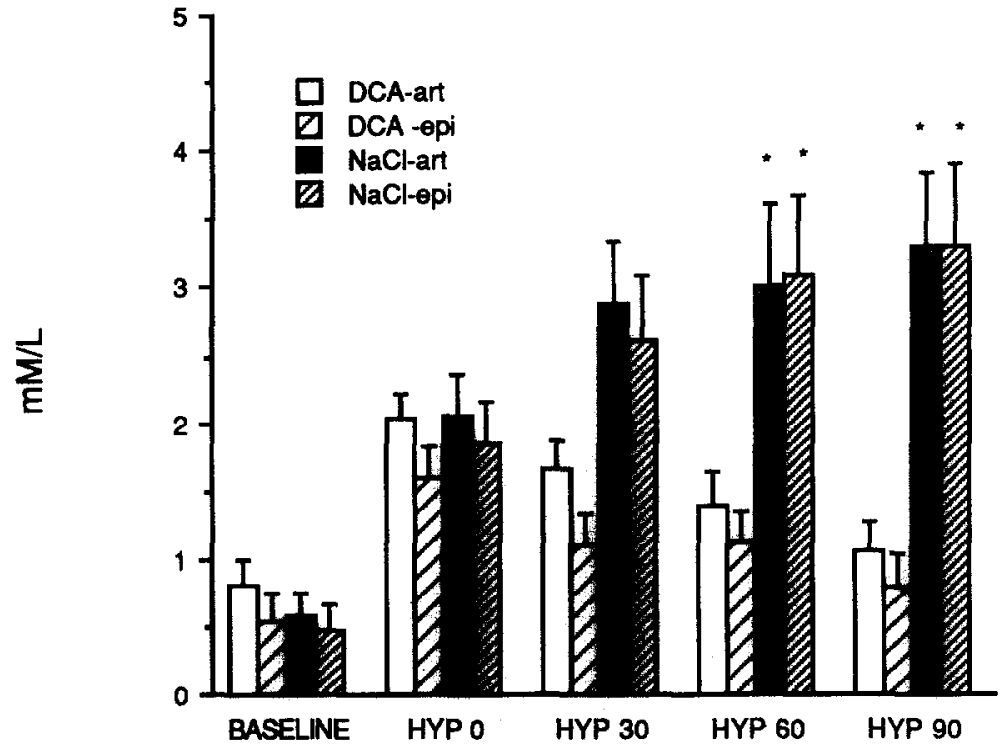

Fig 3. Changes in arterial lactate and epicardial lactate during treatment of hypoxic lactic acidosis with DCA or NaCl. All values are mean \pm SEM $* P<$ 0.01 compared to $\mathrm{NaCl}$. to epicardium. The bodies of the papillary muscles were discarded. Full thickness renal sections were divided into outer and inner cortex and outer and inner medulla. The tissue samples were weighed and placed in counting vials for assay of radioactivity in a Tracor gamma scintillation counter (model 1185, GammaTrac, Elk Grove Village, IL). After correcting the counts in each tissue sample for background and overlapping counts with simultaneous equations, blood flow was calculated with the equation: $\mathrm{Qm}=$ $(\mathrm{Cm} \times \mathrm{Qr}) / \mathrm{Cr}$ where $\mathrm{Qm}=$ myocardial or renal blood flow in $\mathrm{mL} / \mathrm{min}, \mathrm{Cm}=$ counts $/ \mathrm{min}$ in tissue samples, $\mathrm{Qr}=$ withdrawal rate of the reference arterial sample in $\mathrm{mL} / \mathrm{min}$, and $\mathrm{Cr}=$ counts/min in the reference arterial sample. Flow per gram of tissue was calculated by dividing flow by the weight of the appropriate sample. Background and overlap corrections and blood flow calculations were performed on an Apple Ile microcomputer (Cupertino, CA). Hemodynamic, metabolic, and MBF data were analyzed using ANOVA (Scheffe) with $95 \%$ confidence limits; all results are reported as mean \pm standard error.

\section{RESULTS}

A total of 43 animals were entered into this study; 20 deaths occurred due to irreversible shock. Four deaths occurred prior to the hypoxia endpoint, 13 deaths occurred between $\mathrm{H}$ and $\mathrm{H} 60$ ( $7 \mathrm{NaCl}, 6 \mathrm{DCA})$, and 2 deaths between $\mathrm{H} 60$ and $\mathrm{H} 90$ ( $2 \mathrm{NaCl}, 0 \mathrm{DCA})$. The high mortality noted in this model $(46 \%)$ is similar to that seen clinically in postoperative patients with lactic acidosis and reflects the

Table 1. Hemodynamic Effects of DCA in Hypoxic Lactic Acidosis

\begin{tabular}{|c|c|c|c|c|c|c|}
\hline & $N$ & Baseline & HYP 0 & HYP 30 & HYP 60 & HYP 90 \\
\hline \multicolumn{7}{|c|}{ MAP (mmHg) } \\
\hline DCA & 10 & $133 \pm 7.6$ & $159 \pm 10$ & $165 \pm 10^{*}$ & $141 \pm 12$ & $116 \pm 15.0 \dagger$ \\
\hline $\mathrm{NaCl}$ & 11 & $133 \pm 7.3$ & $170 \pm 11$ & $162 \pm 13^{*}$ & $140 \pm 12$ & $123 \pm 17.0 \dagger$ \\
\hline \multicolumn{7}{|c|}{$\mathrm{CO}(\mathrm{L} / \mathrm{min})$} \\
\hline DCA & 10 & $5.35 \pm 0.63$ & $5.40 \pm 0.5$ & $6.19 \pm 0.5$ & $6.36 \pm 0.4$ & $6.95 \pm 0.60$ \\
\hline $\mathrm{NaCl}$ & 11 & $5.19 \pm 0.3$ & $5.97 \pm 0.6$ & $5.95 \pm 0.5$ & $6.14 \pm 0.4$ & $5.27 \pm 0.57$ \\
\hline \multicolumn{7}{|c|}{ LAP (mmHg) } \\
\hline DCA & 10 & $7.6 \pm 1.1$ & $12.8 \pm 2.4$ & $16.0 \pm 3.3$ & $11.9 \pm 1.5$ & $9.2 \pm 2.1$ \\
\hline $\mathrm{NaCl}$ & 11 & $7.5 \pm 0.9$ & $9.6 \pm 1.4$ & $10.0 \pm 1.8$ & $9.7 \pm 2.7$ & $9.3 \pm 2.3$ \\
\hline \multicolumn{7}{|c|}{ SVR (dyne $\cdot \mathrm{sec} \cdot \mathrm{cm}^{-5}$ ) } \\
\hline DCA & 10 & $1987 \pm 160$ & $2510 \pm 330$ & $2266 \pm 317$ & $1872 \pm 263$ & $1361 \pm 247 t$ \\
\hline $\mathrm{NaCl}$ & 11 & $2052 \pm 147$ & $2358 \pm 250$ & $2291 \pm 258$ & $1944 \pm 280$ & $1958 \pm 326$ \\
\hline \multicolumn{7}{|c|}{ HR (beat/min) } \\
\hline DCA & 10 & $134 \pm 5$ & $155 \pm 10$ & $145 \pm 12$ & $159 \pm 16$ & $182 \pm 11$ \\
\hline $\mathrm{NaCl}$ & 11 & $155 \pm 4$ & $144 \pm 4$ & $155 \pm 5$ & $174 \pm 5$ & $189 \pm 5^{*}$ \\
\hline \multicolumn{7}{|l|}{ DPDT } \\
\hline DCA & 10 & $2453 \pm 286$ & $3593 \pm 370^{*}$ & $4460 \pm 354^{*}$ & $4275 \pm 224^{*}$ & $3737 \pm 275^{*}$ \\
\hline $\mathrm{NaCl}$ & 11 & $2782 \pm 190$ & $4688 \pm 450^{*}$ & $4981 \pm 514^{*}$ & $5182 \pm 551^{*}$ & $4250 \pm 615^{*}$ \\
\hline
\end{tabular}

NOTE, All values are means $\pm S E$.

${ }^{*} P \leq 0.01$ compared to CON.

tP $\leq 0.05$ compared to HYP. 
severity of this model and the significance of this metabolic derangement. No difference in severity of acidosis was noted between survivors versus those animals that died.

No differences between groups were found in metabolic measurements at baseline and the severity of lactic acidosis was equivalent at the beginning of treatment (Figs 1-3). There was no difference in $\mathrm{PaO}_{2}, \mathrm{PaCO}_{2}$, or oxygen saturation between groups at any measurement period. The metabolic status of the animals receiving $\mathrm{NaCl}$ continued to significantly deteriorate from HYP 0 to HYP 90, with a significant decrease in pH (Fig 1), bicarbonate (Fig 2), and an increase in both arterial and epicardial lactate (Fig 3), whereas those animals receiving DCA showed stabilization in all metabolic parameters.

Baseline arterial and epicardial lactates for both groups ranged from 0.47 to $0.81 \mathrm{mmol} / \mathrm{L}$ and showed a threefold to fourfold increase at the attainment of hypoxic acidosis (HYP 0). Lactate levels were determined on whole blood samples using an enzymatic one-step technique, whereas the more commonly reported colorometric method requires multiple steps and reports plasma levels. Colorometrically determined plasma levels range from 1 to $1.5 \mathrm{mM} / \mathrm{L}$ during normal conditions with levels increasing to $5 \mathrm{mM} / \mathrm{L}$ or greater during lactic acidosis. The magnitude of the increase in lactate during hypoxia and subsequent alterations during treatment in this study concur with those reported elsewhere. The variance between these results and thuse of others most likely reflects the difference in this new, simpler technique.

Hemodynamic results are shown in Table 1. Both groups showed equivalent hemodynamic parameters at baseline and had a similar hemodynamic response to hypoxia. Cardiac output and stroke volume were significantly increased at HYP 90 in those animals receiving DCA, but not in those receiving $\mathrm{NaCl}$. The first derivative of developed pressure $(\mathrm{dP} / \mathrm{dt})$ was dramatically increased in both groups by hypoxia, but was not further altered by treatment with DCA or $\mathrm{NaCl}$. However, while MAP decreased similarly from HYP 0 to HYP 90 in both groups, SVR decreased in DCA dogs but did not change in those receiving $\mathrm{NaCl}$.

Myocardial blood flow (Fig 4) increased dramatically in all regions of the heart in both groups in response to hypoxia and was not further altered by treatment with DCA or $\mathrm{NaCl}$. Neither myocardial oxygen delivery (milliliters of $\mathrm{O}_{2} / \mathrm{g}$ of tissue/min) nor myocardial oxygen consumption was different between groups, but myocardial oxygen use efficiency ( $\mathrm{LV}$ work/ $\mathrm{MVO}_{2}$ ) improved from HYP 0 to HYP 90 in those animals receiving DCA versus those receiving $\mathrm{NaCl}$ (Fig 5).

Total renal blood flow did not increase in either group from baseline at HYP 0 , but renal cortical blood flow did increase from HYP 0 to HYP 90 in the DCA-treated dogs. There was no change in renal medullary blood flow in either group. Serum sodium increased similarly in both groups during treatment, while urine production and sodium diuresis [(urine sodium)(volume)/time] was greater at HYP 90 in the DCA treated group when compared to those receiving $\mathrm{NaCl}$ (Fig 6).
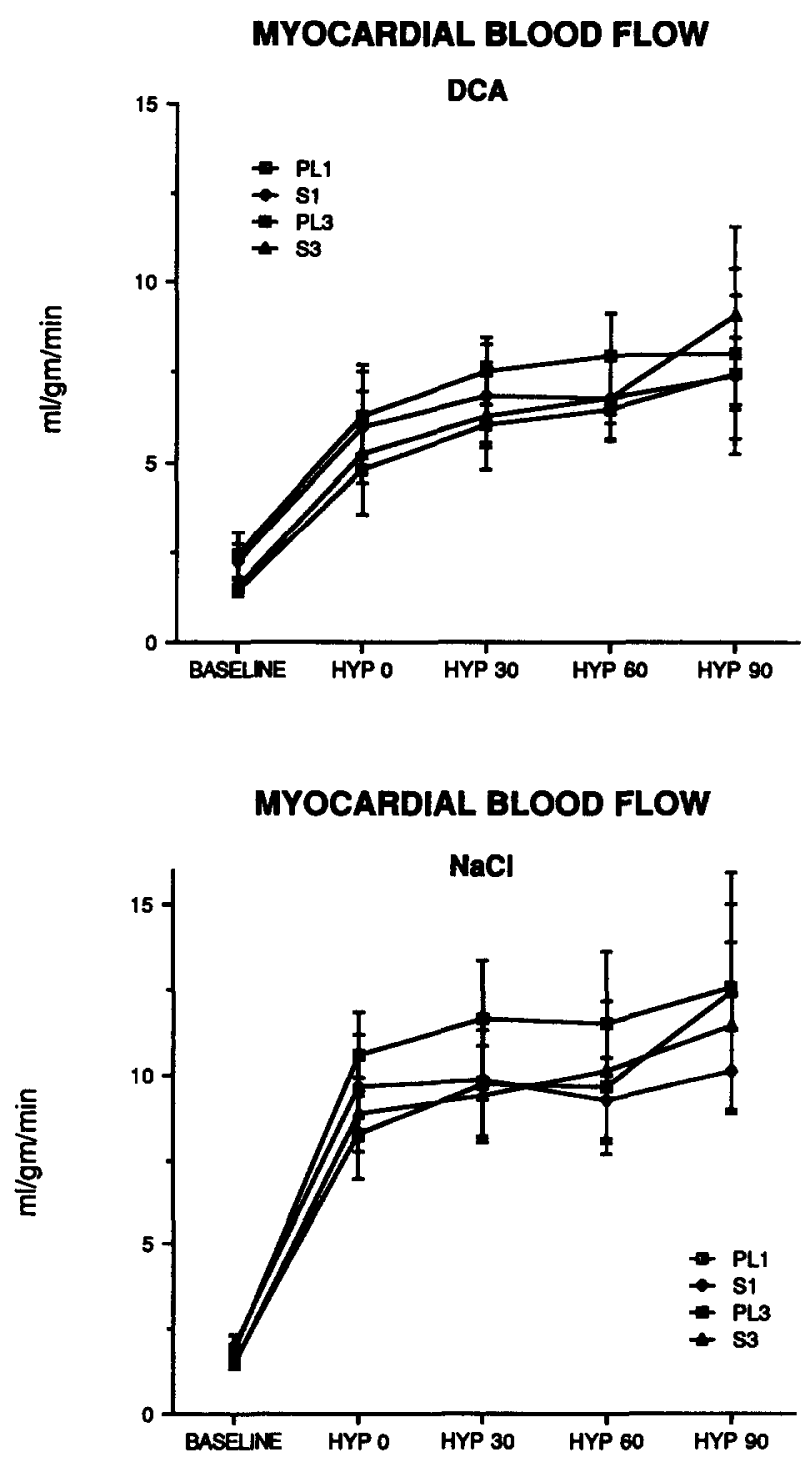

Fig 4. Changes in myocardial blood fiow during treatment of hypoxic lactic acidonis with DCA or NaCL. Abbreviations: PL1, posterolateral eplcardium; S1, septal epicardium; PL3, posterolateral ondocardium; S3, septal endocardlum.

\section{DISCUSSION}

These results of the hemodynamic and metabolic effects of DCA in this canine model of induced hypoxic lactic acidosis demonstrate that DCA administration results in a dramatic improvement in metabolic status and enhanced myocardial performance by altering myocardial efficiency. The lowering of whole blood levels of lactate and improvement in $\mathrm{pH}$ and bicarbonate in this model precedes the improvement in hemodynamic status and probably reflects a primary metabolic effect of DCA. In this model the worsening acidosis of the control dogs resulted in peripheral vasoconstriction and poor cardiac performance, while DCA enhanced myocardial performance without increasing the "cost" $\left(\mathrm{MVO}_{2}\right.$, contractility or blood flow) of myocardial work.

These results are similar to those reported by Graf, who 


\section{PERCENT CHANGE FROM HYPOXIA}

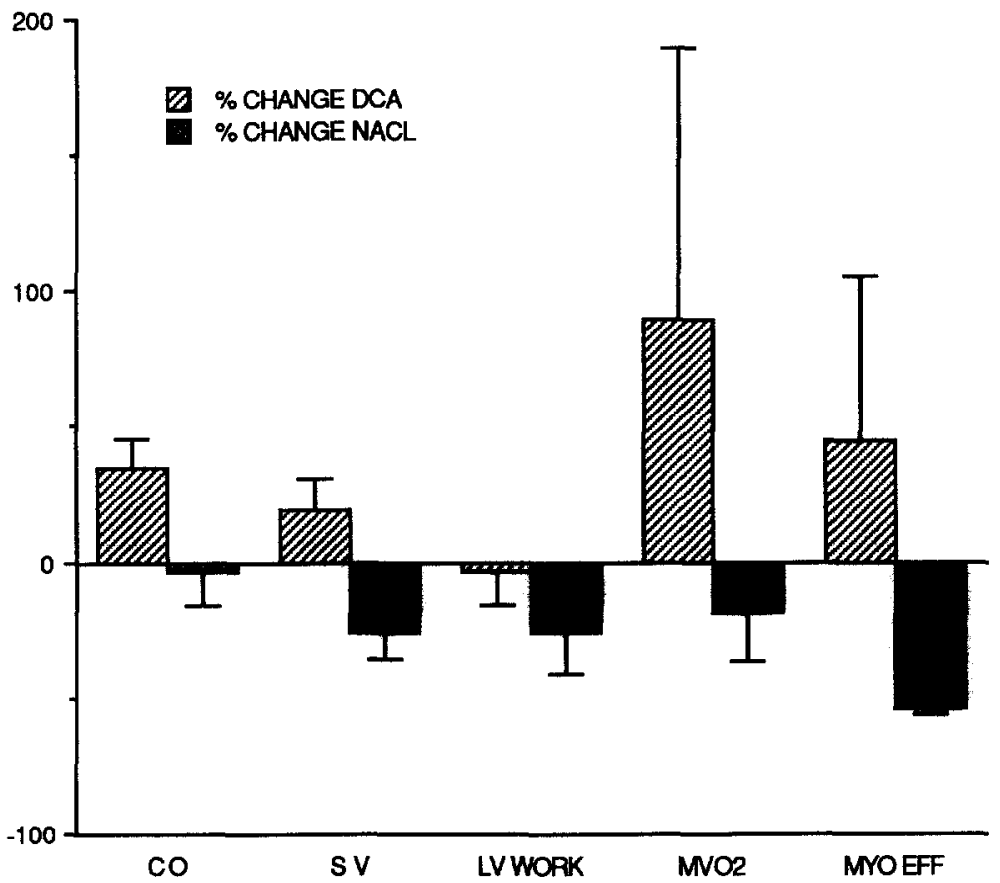

Fig 5. Percent change in myocardial work indices from HYP 0 to HYP 90 during treatment with DCA or $\mathrm{NaCl}$. Abbreviations: CO, cardiac output; SV, stroke volume: $\mathrm{LV}$ Work, left ventricular work; $\mathrm{MVO}_{2}$, myocardial oxygen consumption (global); MYO EFF, myocardial efficiency, ie, left ventricular work per millititer of oxygen consumption. found that treatment of canine hypoxic lactic acidosis with DCA improved metabolic parameters without changing blood pressure or cardiac index. ${ }^{11}$ They did not investigate myocardial blood flow or contractility, but did show that metabolic hepatic lactate extraction was increased with DCA compared to $\mathrm{NaCl}$ treatment. DCA treatment of hypoxic lactic acidosis in rats resulted in increased blood pressure as well as higher $\mathrm{pH}$ and bicarbonate. ${ }^{12}$ Whereas an improvement in CO with DCA was noted, no change in blood pressure was found. This may be due to a species

\section{SODIUM DIURESIS}

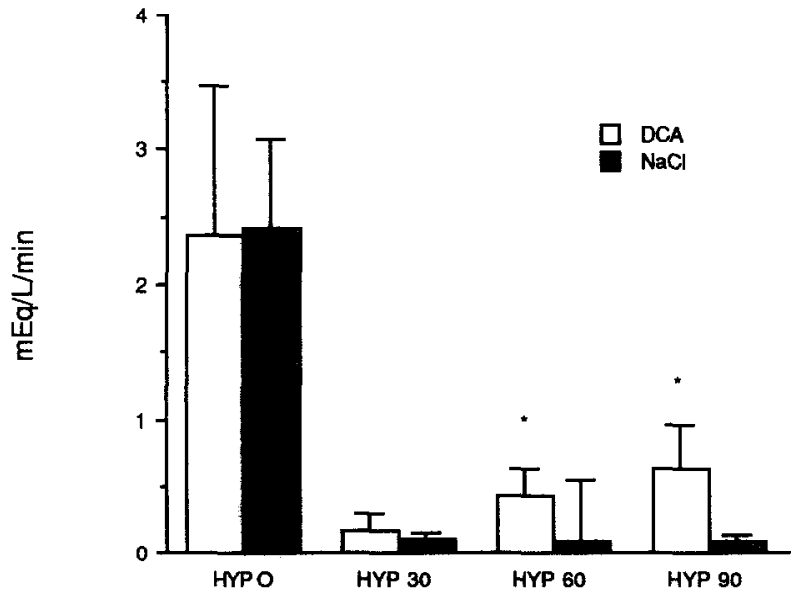

Fig 6. Sodium diuresis during treatment of hypoxic lactic acidosis with DCA or $\mathrm{NaCl}$. All values are means \pm SEM. $* P<0.01$ compared to $\mathrm{NaCl}$. difference, because hypoxia in the rat model resulted in stable hypotension, but in the model dogs who developed hypotension related to hypoxia died within 5 to 10 minutes of irreversible cardiac failure. Clinically, hypotensive patients with severe lactic acidosis treated with DCA showed an improvement in blood pressure as well as $\mathrm{pH}$ and bicarbonate, but these reports do not report hemodynamics or any indices of oxygen uptake or delivery. 13,14

Previous reports of the effect of DCA on myocardial performance and metabolism include both laboratory and clinical studies. DCA has been shown to limit ST segment elevation, increase glucose extraction and decrease myocardial lactate release in dogs with acute myocardial ischemia due to abrupt coronary occlusion. ${ }^{15}$ In addition, DCA has been shown to enhance the inotropic effect of amrinone and ouabain, and stimulate glucose oxidation in isolatcd working hearts from endotoxin shocked rats. ${ }^{16}$ In this model, DCA treatment increased cardiac output, stroke volume, and decreased SVR, but did not alter $\mathrm{dP} / \mathrm{dt}$ compared to $\mathrm{NaCl}$ treatment. Specific upregulation of inotropy with DCA treatment was not shown although the tremendous increase in $\mathrm{dP} / \mathrm{dt}$ occurring in both groups in response to severe hypoxia may have masked such an effect. However, an improvement in the amount of myocardial work done per oxygen consumed during DCA administration (Fig 4) was found, similar to a study of 9 patients with coronary artery disease, in whom DCA administration increased stoke volume, decreased SVR, and enhanced myocardial efficiency index ( $\mathrm{LV}$ work $\left./ \mathrm{MVO}_{2}\right){ }^{17}$

The mechanism of DCA's beneficial metabolic effect may relate to DCA's ability to stimulate the activity of pyruvate dehydrogenase (PDH), the multienzyme complex respon- 
sible for oxidation of pyruvate to acetyl CoA. ${ }^{19,20}$ During normal cellular metabolism pyruvate is produced by glycolysis and subsequently is either oxidized to acetyl CoA and enters the Krebs cycle, or is metabolized to lactate. The use of pyruvate in the Krebs cycle, when coupled to oxidative phosphorylation and the electron transport chain, provides the majority of cellular energy requirements, but is dependent on an adequate supply of oxygen. As oxygen delivery decreases, electron flux through the electron transport chain slows, ATP production decreases, and NADH can no longer be converted to NAD ${ }^{+}$. This decrease in ATP stores directly enhances anaerobic glycolysis, which increases pyruvate production, and the $\mathrm{NAD}^{+}$required for this anaerobic glycolysis is supplied during the formation of lactate from pyruvate. The increase in NADH levels that occurs during hypoxia inhibits PDH enzyme activity, decreasing entry of pyruvate into the Krebs cycle and increasing its transformation to lactate. Finally, the normal pathway for consumption of lactate, gluconeogenesis in the liver and kidney, is inhibited by hypoxia. It is apparent how tissue hypoxia, with increased production and decreased use of pyruvate/lactate quickly results in lactic acidosis. By directly increasing the activity of $\mathrm{PDH}, \mathrm{DCA}$ appears to override the negative feedback loop of NADH accumulation, increasing the amount of pyruvate metabolized to acetyl $\mathrm{CoA}$ and decreasing the production of lactate.

The hemodynamic improvement noted may be related to this enhanced entry of pyruvate into the Krebs cycle. These results indicate a change in energy metabolism (increased myocardial efficiency) and a change in acid base balance (improvement in $\mathrm{pH}$ and bicarbonate) with DCA treatment in hypoxic lactic acidosis. Although it can be speculated which effect is primarily responsible for the improvement in hemodynamic parameters noted, further work on the myocardial and cellular effects of DCA will be required to delineate the mechanism. In conclusion, DCA can effectively ameliorate the adverse metabolic effects of hypoxic acidosis, preventing further decreases in serum $\mathrm{pH}$ and bicarbonate or further increases in blood lactate levels. DCA appears to enhance myocardial performance without a direct inotropic effect, increasing stroke volume and thus cardiac output, improving myocardial oxygen use efficiency.

\section{REFERENCES}

1. Blair E, Cowley RA, Tait MK: Refractory septic shock in man: Role of lactate and pyruvate metabolism and acid-base balance in prognosis. Ann Surg 31:537-540, 1965

2. Buchalter SE, Crain MR, Kreisberg RA: Regulation of lactate metabolism in vivo. Diabetes/Met Rev 5:379-391, 1989

3. Olivia PB: Lactic acidosis. Am J Med 48:209-225, 1970

4. Kreisberg RA: Pathogenesis and management of lactic acidosis. Ann Rev Med 35:181-193, 1984

5. Bersin RM, Arieff AI: Improved hemodynamic function during hypoxia with Cardicarb, a new agent for the management of acidosis. Circ 77:277-233, 1988

6. Graf $\mathrm{H}$, Leach W, Arieff AI: Metabolic effects of sodium bicarbonate in hypoxic lactic acidosis in dogs. Am $\mathrm{J}$ Physiol 249:F630-F635, 1985

7. Grekin RJ, Tannen RL: Ketoacidosis, hyperosmolar states, and lactic acidosis, in Kokko JP, Tannen RL (eds). Fluids and Electrolytes. Philadelphia, PA, Saunders, 1990:873-905.

8. Stacpoole PW, Moore GW, Kornhauser DM: Metabolic effects of dichloroacetate in patients with diabetes mellitus and hyperlipoproteinemia. N Engl J Med 298:526-630, 1978

9. Park R, Arieff AI: Treatment of lactic acidosis with dichloroacetate in dogs. J Clin Invest 70:853-862, 1982

10. Merrill GF, Zambraski EJ, Grassl SM: Effect of dichloroacetate on plasma lactic acid in exercising dogs. J Appl Physiol 48:427-431, 1980

11. Graf H, Leach W, Arieff AI: Effects of dichloroacetate in the treatment of hypoxic lactic acidosis in dogs. J Clin Invest 76:919-923, 1985
12. Romeh SA, Tannen RL: Therapeutic benefit of dichloroacetate in experimentally induced hypoxic lactic acidosis. J Lab Clin Med 107:378-383, 1986

13. Stacpoole PW, Harman EM, Curry SH, Baumgartner TG, Misbin RI: Treatment of lactic acidosis with dichloroacetate. N Engl J Med 309:390-396, 1983

14. Stacpoole PW, Lorenz AC, Thomas RG, Harman EM: Dichloroacetate in the treatment of lactic acidosis. Ann Int Med 108:58-63, 1988

15. Mjos OD, Miller NE, Riemersma RA, Oliver MF: Effects of dichloroacetate on myocardial substrate extraction, epicardial ST-segment elevation, and ventricular blood flow following coronary occlusion in dogs. Cardiov Res 10:427-436, 1976

16. Burns AH, Summer WR, Burns LAR, Shepherd RE: Inotropic interactions of dichloroacetate with amrinone and ouabain in isolated hearts from endotoxin-shocked rats. J Cardiov Pharmacol 2:379-386, 1988

17. Wargovich TJ, MacDonald RG, Hill JA, Feldman RL, Stacpoole PW, Pepine CJ: Myocardial metabolic and hemodynamic effects of dichloroacetate in coronary artery disease. Am J Cardiol 61:65-70, 1988

18. Heymann MA, Payne BD, Hoffman JE, Rudolph AN: Blood flow measurements with radionuclide-labeled particles. Prog Cardiovasc Dis 10:55-79, 1977

19. Whitehouse S, Cooper RH, Randle PJ: Mechanism of activation of pyruvate dehydrogenase by dichloroacetate and other halogenated carboxylic acids. Biochem J 142:761-774, 1974

20. Stacpoole PW: The pharmacology of dichloroacetate. Metabolism 38:1124-1144, 1989 